\title{
Ground states of dispersion-managed nonlinear Schrödinger equation
}

\author{
Vadim Zharnitsky* \\ Division of Applied Mathematics, Brown University, Providence, Rhode Island 02912 \\ Emmanuel Grenier \\ UMPA, Ecole Normale Superiéure de Lyon, 46 Allée d'Italie, 69364 Lyon Cedex 7, France \\ Sergei K. Turitsyn \\ Photonics Research Group, School of Engineering and Applied Science, Aston University, Birmingham B4 7ET, United Kingdom
}

Christopher K. R. T. Jones and Jan S. Hesthaven

Division of Applied Mathematics, Brown University, Providence, Rhode Island 02912

(Received 6 October 1999; revised manuscript received 26 June 2000)

\begin{abstract}
An exact pulse for the parametrically forced nonlinear Schrödinger equation (NLS) is isolated. The equation governs wave envelope propagation in dispersion-managed fiber lines with positive residual dispersion. The pulse is obtained as a ground state of an averaged variational principle associated with the equation governing pulse dynamics. The solutions of the averaged and original equations are shown to stay close for a sufficiently long time. A properly adjusted pulse will therefore exhibit nearly periodic behavior in the time interval of validity of the averaging procedure. Furthermore, we show that periodic variation of dispersion can stabilize spatial solitons in a Kerr medium and one-dimensional solitons in the NLS with quintic nonlinearity. The results are confirmed by numerical simulations.
\end{abstract}

PACS number(s): 42.65.Tg, 05.45.Yv

\section{INTRODUCTION}

Recent technological advances in material science have afforded considerable freedom in the design of optical materials. In particular, an optical medium can be designed to have variable group velocity dispersion, nonlinearity, and polarization [1]. All of these parameters can be varied on different scales by using appropriate manufacturing processes. It is especially convenient to produce materials/ systems having physical parameters varying periodically, as this amounts to a repetitive manufacturing process. The evolution equations guiding the propagation of electromagnetic pulses (or their envelopes) in such systems are nonlinear evolution equations with periodically varying coefficients. Although such systems are frequently encountered in applications, there are few general techniques for analyzing pulse dynamics in parametrically forced systems.

The clearest realization of this technology can be found in the field of fiber-optic communication, namely, in the socalled dispersion-managed (DM) optical data transmission that was proposed in 1980 [2]. The simplest optical-pulse equalizing system consists of a transmission fiber and an equalizer fiber with the opposite dispersion. The periodic incorporation of a compensating fiber reduces (or even eliminates) the total dispersion of the fiber spans. In the linear regime, the compensation of dispersion aims to prevent dispersive broadening of the pulse. In the nonlinear regime, an additional advantage is that the impact of four-wave mixing on a signal transmission is substantially suppressed due to

\footnotetext{
*Present address: Mathematical Sciences Research, Lucent Technologies, 600 Mountain Ave., 2C-359, Murray Hill, NJ 07974.
}

the reduction of the efficiency of phase matching. The practical achievements of this approach have stimulated further study of nonlinear dynamics in media with varying coefficients.

In spite of the practical advances in the realization of such systems, some basic features of a DM signal are not fully understood. In this article, we show the existence and stability of the DM soliton in one important special case (positive residual dispersion). In the case of vanishing residual dispersion, we verify some nontrivial conditions for stability, one of which is the boundedness from below of the Hamiltonian functional. It turns out to be bounded entirely due to a subtle smoothing effect resulting from the variable dispersion parameter. Indeed, the large rapid variation of dispersion makes the pulse undergo rapid oscillations, which smooth out the peaks that could cause instability.

Having understood and properly formulated this effect, which one might call dispersive smoothing, we then show that in some other systems of practical importance stable ground states can be created.

\section{DISPERSION-MANAGED SOLITON AS A GROUND STATE OF THE AVERAGED VARIATIONAL PRINCIPLE}

We start from the wave envelope equation, derived from the Maxwell equations, guiding the propagation of electromagnetic pulses in optical fibers. We show that the averaged equation possesses a ground state minimizing an averaged action functional. The corresponding solutions of the original equation are constructed from this ground state and turn out to be nearly periodic for sufficiently long times (while the averaging procedure remains valid).

After nondimensionalizing and rescaling in the strong dis- 
persion management regime the wave envelope propagation equation takes the form (see, e.g., [3])

$$
i u_{z}+d(z) u_{\tau \tau}+\epsilon\left(|u|^{2} u+\alpha u_{\tau \tau}\right)=0
$$

where $u$ is the complex amplitude of the electric field, $z$ is the propagation distance, $\tau$ is the retarded time, $d(z+1)=d(z)$ is the mean-zero component of the group velocity dispersion, and $\epsilon\langle d\rangle$ is the residual dispersion. The $\epsilon$ smallness of the mean dispersion corresponds to the so-called nonweak dispersion management regime. We first derive the slowly varying Hamiltonian and introduce the averaged Hamiltonian [4].

The above equation possesses a Hamiltonian functional similar to that of the nonlinear Schrödinger equation (NLS) [4]

$$
H=\int_{-\infty}^{+\infty}\left[d(z)\left|u_{\tau}\right|^{2}+\epsilon\left(\alpha\left|u_{\tau}\right|^{2}-\frac{1}{2}|u|^{4}\right)\right] d \tau .
$$

Solving the unperturbed equation (with $\epsilon=0$ ), we obtain $u(\tau, z)=T(z) u(\tau, 0)$, where $T(z)$ is the fundamental solution operator of $i u_{z}+d(z) u_{\tau \tau}=0$.

The family of unitary operators $T(z)$ is periodic $T(z$ $+1)=T(z)$ since $\langle d(z)\rangle=0$. Using the solution of the linear system according to the method of variation of constants, we introduce a canonical transformation $u(z, \tau)=T(z) v(z, \tau)$. The new Hamiltonian takes the form

$$
H=\epsilon \int_{-\infty}^{+\infty}\left(\alpha\left|v_{\tau}\right|^{2}-\frac{1}{2}|T(z) v|^{4}\right) d \tau
$$

with the corresponding Euler-Lagrange equation

$$
i v_{z}+\epsilon \alpha v_{\tau \tau}+\epsilon T^{-1}(z)\left(|T(z) v|^{2} T(z) v\right)=0 .
$$

Now we turn to the averaged variational principle

$$
\langle H\rangle=\epsilon \int_{-\infty}^{+\infty} \int_{0}^{1}\left(\alpha\left|v_{\tau}\right|^{2}-\frac{1}{2}|T(z) v|^{4}\right) d \tau d z
$$

with the corresponding averaged equation, previously derived in [4],

$$
i v_{z}+\epsilon \alpha v_{\tau \tau}+\epsilon \int_{0}^{1} T^{-1}(z)\left(|T(z) v|^{2} T(z) v\right) d z=0 .
$$

The solutions of the averaged equation are close to the solutions of the original equation in the following sense. In the interval $0 \leqslant z \leqslant C \epsilon^{-1}$, there exists a solution of the averaged equation (2) that has an algebraic decay in Fourier space,

$$
\int_{-\infty}^{+\infty}|\hat{v}(z, k)|^{2}\left(1+k^{2}\right)^{s} \leqslant C \text { if } \int_{-\infty}^{+\infty}|\hat{v}(0, k)|^{2}\left(1+k^{2}\right)^{s} \leqslant C,
$$

where $\hat{v}(z, k)$ is the Fourier transform of $v(z, \tau)$.

Furthermore, the solution $\tilde{v}(z, \tau)$ of the full equation (1), with the same initial data $\tilde{v}(0, \tau)=v(0, \tau)$, is close to the solution of the averaged equation, in the sense that

$$
\int_{-\infty}^{+\infty}|\hat{v}(z, k)-\hat{\widetilde{v}}(z, k)|^{2}\left(1+k^{2}\right)^{s-3} d k \leqslant C \epsilon
$$

for $0 \leqslant z \leqslant C \epsilon^{-1}$. Establishing these estimates requires a careful study of the averaging procedure developed in [5], and by one of the authors in [6]. The details of the averaging procedure will appear elsewhere [7].

Below we show that the averaged equation possesses a family of ground state solutions, which together with the averaging result verify the existence of nearly periodic, stable pulses.

It is easy to see that Eq. (2) is also phase invariant and therefore has a conserved quantity

$$
P(v)=\int_{-\infty}^{+\infty}|v|^{2} d \tau
$$

Introducing another useful functional corresponding to a Sobolev norm,

$$
G(v)=\int_{-\infty}^{+\infty}\left(|v|^{2}+\left|\frac{\partial v}{\partial \tau}\right|^{2}\right) d \tau,
$$

we consider the constrained minimization problem

$$
P_{\lambda}=\inf \{E(v)=\langle H\rangle(v), G(v)<\infty, P(v)=\lambda\} .
$$

If there is a solution of this problem $u(x)$ then it corresponds to a standing wave solution of the full averaged equation (2). Although $P_{\lambda}$ is bounded from below (as we will show later), it does not guarantee the presence of a minimizer as simple examples show. Therefore, folowing the standard approach, we construct a minimizing sequence that converges to a minimizer.

Note that $P_{\lambda} \leqslant 0$, since the sequence of vanishing Gaussians

$$
v_{k}(\tau)=\frac{1}{\sqrt{4 \pi k / \lambda}} \exp \left(-\frac{\tau^{2}}{4 k}\right)
$$

satisfies the constraint and $E\left(v_{k}\right) \rightarrow 0$ as $k \rightarrow \infty$. This can be checked by direct calculations using the well known fact that Gaussian functions are self-similar solutions of the linear Schrödinger equation.

The idea, now, is to find a minimizing sequence $v_{k}$ $\left[P(v)=\lambda, E\left(v_{k}\right) \rightarrow P_{\lambda}\right]$ such that $v_{k} \rightarrow v$, which would be a minimizer satisfying the Euler-Lagrange equation. First, we must show that $-\infty<P_{\lambda}<0$. The first part of the inequality is required so that a hypothetical minimizer can satisfy the Euler-Lagrange equation. The second inequality $\left(P_{\lambda} \neq 0\right)$ is necessary to avoid minimizing sequences converging to $v$ $\equiv 0$, as in the above example.

By integrating an inequality of Sobolev type over $z$,

$$
\begin{aligned}
\int|T(z) v|^{4} d \tau & \leqslant \frac{1}{\sqrt{3}}\left(\int\left|T v_{\tau}\right|^{2} d \tau\right)^{1 / 2}\left(\int|T v|^{2} d \tau\right)^{3 / 2} \\
& =\frac{1}{\sqrt{3}}\left(\int\left|v_{\tau}\right|^{2} d \tau\right)^{1 / 2}\left(\int|v|^{2} d \tau\right)^{3 / 2} \\
& =\frac{1}{\sqrt{3}} \lambda^{3 / 2} P\left(v_{\tau}\right)^{1 / 2}
\end{aligned}
$$


we obtain $[8]$

$$
E(v) \geqslant P\left(v_{\tau}\right)-\frac{1}{\sqrt{3}} \lambda^{3 / 2} P\left(v_{\tau}\right)^{1 / 2} \geqslant-\frac{\lambda^{3}}{12} .
$$

Using self-similar solutions of the linear equation corresponding to Gaussian pulses, we show by straightforward calculations (see the Appendix) that $P_{\lambda}<0$ for any $\lambda>0$. Note that for any minimizing sequence $G\left(v_{k}\right)$ is bounded, for otherwise $E\left(v_{k}\right)$ would have infinitely many positive terms by the above inequality.

Next, we show that for any minimizing sequence $v_{k}$ there exists a subsequence $v_{k_{m}}$ and a real number $\tau_{m}$ such that $w_{m}(\tau)=v\left(\tau-\tau_{m}\right)$ converges to a pulselike periodic solution $v$. First, applying Lions' concentration compactness principle (see the Appendix), we find that $w_{m} \rightarrow v$ so that $P\left(w_{m}-v\right) \rightarrow 0$ and $P(v)=\lambda$ (the limit satisfies the constraint). Since $G\left(w_{m}\right)<\infty$ there exists a subsequence also denoted $w_{m}$ weakly converging to $v$.

Following the well known procedure (see [9]) it is possible to show that $G\left(w_{m}-v\right) \rightarrow 0$, which implies that the minimizer possesses a weak derivative. Since both functionals $\langle H\rangle(v)$ and $P(v)$ are of class $C^{1}$ then, for some Lagrangian multiplier $\omega$, the obtained minimizer $v$ weakly satisfies the Euler-Lagrange equation (2) corresponding to the averaged variational principle, i.e.,

$$
\omega v+\epsilon \alpha v_{\tau \tau}+\epsilon \int_{0}^{1} T^{-1}(z)\left(|T(z) v|^{2} T(z) v\right)=0,
$$

thus corresponding to a standing wave solution $e^{-i \omega z} v(\tau)$ of the averaged equation (2).

Using the classical bootstrapping argument, we obtain the result that the minimizer has superalgebraic decay in Fourier space,

$$
\int_{-\infty}^{+\infty}|\hat{v}(k)|^{2}\left(1+k^{2}\right)^{s} d k<C \text { for any } s,
$$

and thus it is smooth. Now we can construct a set of initial data which behave nearly periodically. We take our initial data $\hat{v}(k, 0)$ close to the ground state solution, which we will denote by $v_{g}(k)$,

$$
\int_{-\infty}^{+\infty}\left|\hat{v}_{g}(k)-\hat{v}(k)\right|^{2}\left(1+k^{2}\right)^{4} d k<C \epsilon,
$$

and therefore, by the local existence theorem, the corresponding solutions $\hat{v}_{g}(k, z)$ and $\hat{v}(k, z)$ of the averaged equation (2) stay $\epsilon$-close in the same norm. On the other hand, we can apply the averaging result to conclude that the solutions $\hat{v}(k, z)$ and $\tilde{\hat{v}}(k, z)$ of the averaged (2) and the original (1) equations stay close for $0 \leqslant z \leqslant C \epsilon^{-1}$,

$$
\int_{-\infty}^{+\infty}|\tilde{\hat{v}}(k)-\hat{v}(k)|^{2}\left(1+k^{2}\right) d k<C \epsilon .
$$

Therefore, a solution initially close to a ground state will stay near it,

$$
\int_{-\infty}^{+\infty}\left|\tilde{\hat{v}}(k, z)-\hat{v}_{g}(k, z)\right|^{2}\left(1+k^{2}\right) d k<C \epsilon,
$$

while $z \leqslant C \epsilon^{-1}$. Thus, we have shown that an initial pulse with sufficiently fast decay in Fourier space and close to the ground state of the averaged variational principle will stay nearly periodic for $z \leqslant C \epsilon^{-1}$.

\section{GROUND STATES IN THE CASE OF ZERO AND NEGATIVE RESIDUAL DISPERSION}

In the mean-zero average dispersion case, one does not expect ground states in the absence of variable dispersion. The main reason is unboundedness of the Hamiltonian from below. However, due to the smoothing properties of the linear Schrödinger equation the Hamiltonian functional is bounded from below. Formally, this follows from the Strichartz estimate

$$
\int_{-\infty}^{+\infty} \int_{-\infty}^{+\infty}|u(z, \tau)|^{6} d z d \tau \leqslant C[P(u)]^{1 / 3},
$$

where $u(z, t)$ is the solution of the free Schrödinger equation

$$
i u_{z}+u_{\tau \tau}=0 .
$$

Indeed,

$$
\begin{aligned}
& \int_{0}^{1} \int_{-\infty}^{+\infty}|T(z) u|^{4} d z d \tau \\
& \quad \leqslant \int_{0}^{1} \int_{-\infty}^{+\infty}\left[|T(z) u|^{2}+|T(z) u|^{6}\right] d z d \tau \\
& \quad \leqslant \int_{0}^{1} \int_{-\infty}^{+\infty}|T(z) u|^{2} d z d \tau+\int_{0}^{1} \int_{-\infty}^{+\infty}|T(z) u|^{6} d z d \tau \\
& \quad \leqslant P(u)+C[P(u)]^{1 / 3},
\end{aligned}
$$

where we have used the Strichartz estimate and the conservation of power. Therefore the infimum in the constrained minimization problem

$P_{\lambda}=\left\{E(v)=-\int_{0}^{1} \int_{-\infty}^{+\infty}|T(z) u|^{4} z \tau d z, P(v)<\infty, P(v)=\lambda\right\}$

is bounded from below. Numerous numerical simulations showed that there exists a stable pulse in the mean-zero case. Although we have not been able to establish the presence of a ground state (it would require constructing a converging subsequence), we show that the Hamiltonian functional can be minimized over Gaussian pulses, giving a pulse profile that exhibits stable behavior. Moreover, in the next section we use the above smoothing effect to stabilize pulses in focusing a NLS with critical nonlinearities.

In the case of negative residual dispersion, one should observe instability due to the presence of the negative gradient term. In [7] it is proved that the corresponding constrained variational problem cannot have local minima. Although in numerical experiments stable pulse propagation is observed, it appears to be due to the smallness of the nega- 
tive residual dispersion. Actually, if the value of residual dispersion is decreased further, the stable pulse disappears.

\section{GROUND STATES IN CRITICAL CASES}

Using the above observations, we demonstrate the possibility of stabilization of a spatial pulse in a nonlinear Kerr medium by introducing rapidly varying dispersion. This is reminiscent of Kapitza's phenomenon of the stabilization of the inverted pendulum by rapidly oscillating the pivot [10], as in both cases the stationary states are unstable in the absence of parametric excitation.

The pulse evolution is governed by the two-dimensional nonlinear Schrödinger equation

$$
i u_{Z}+\nabla_{\perp}^{2} u+|u|^{2} u=0,
$$

which is well known to have no stable pulses [9]. We modify the dispersion coefficient by adding a rapidly varying term as in the dispersion management regime,

$$
i u_{Z}+\left[1+\frac{1}{\epsilon} d\left(\frac{Z}{\epsilon}\right)\right] \nabla_{\perp}^{2} u+|u|^{2} u=0
$$

Rescaling the distance $Z=\epsilon z$, we obtain

$$
i u_{z}+d(z) \nabla_{\perp}^{2} u+\epsilon\left(\nabla_{\perp}^{2} u+|u|^{2} u\right)=0
$$

with the Hamiltonian

$$
\begin{aligned}
H(u)= & \int_{-\infty}^{+\infty} \int_{-\infty}^{+\infty}\left(d(z)\left|\nabla_{\perp} u\right|^{2}\right. \\
& \left.-\epsilon\left(\left|\nabla_{\perp} u\right|^{2}-\frac{1}{2}|u|^{4}\right)\right) d x d y .
\end{aligned}
$$

Solving the leading order equation

$$
i u_{z}+d(z) \nabla_{\perp}^{2} u=0,
$$

we use its solutions as the new variable $u(x, y)$ $=T(z) w(x, y)$, where $T(z) w(x, y)$ is the solution of the above equation with initial pulse $w(x, y)$. Carrying out this transformation we obtain the equation for the slowly varying field

$$
i w_{z}+\epsilon \nabla_{\perp}^{2} w+\epsilon Q(w, w, w, z)=0
$$

with the Hamiltonian

$$
H(w)=\epsilon \int_{-\infty}^{+\infty} \int_{-\infty}^{+\infty}\left(\left|\nabla_{\perp} w\right|^{2}-\frac{1}{2}|T(z) w|^{4}\right) d x d y .
$$

Up to this point, we have made no approximations. We now average the Hamiltonian over the fast scale $z$,

$$
\langle H\rangle(w)=\epsilon \int_{0}^{1} \int_{-\infty}^{+\infty} \int_{-\infty}^{+\infty}\left(\left|\nabla_{\perp} w\right|^{2}-\frac{1}{2}|T(z) w|^{4}\right) d x d y d z .
$$

The averaging result stated in the previous section applies in this situation as well (see [7]).
As before, in the averaged evolution problem, the averaged Hamiltonian as well as the power of the pulse is a conserved quantity. Following the above procedure, we fix the power

$$
P(w)=\int_{-\infty}^{+\infty} \int_{-\infty}^{+\infty}|w|^{2} d x d y
$$

and consider the minimization problem

$$
\begin{gathered}
E(P)=\inf \{E(w)=\langle H\rangle(w), \\
\iint\left(\left|\nabla_{\perp} w\right|^{2}+|w|^{2}\right) d x d y<\infty, \\
P=\int|w|^{2} d x d y .
\end{gathered}
$$

The detailed mathematical analysis of this problem is presented in [6]. Here we provide the result: if $P$ is larger than a certain threshold $P_{\text {cr }}$ then there is a pulse minimizing the averaged Hamiltonian.

From the analytical point of view, such a pulse exists because the averaged Hamiltonian is bounded from below $[-\infty<E(P)<0]$. This is in contrast to the constant dispersion regime, where the above minimization problem assumes either zero $[E(P)=0]$ or negative infinity $[E(P)=-\infty]$, either of which excludes the possibility of existence of stable ground states. (Note the similarity with the mean-zero residual dispersion case in a one-dimensional NLS.)

Informally speaking, such boundedness results from the smoothing of the pulse by high local dispersion, which arrests collapse (in the averaged equation) and creates favorable conditions for the existence of ground states. Formally, this stabilizing mechanism is again justified by the corresponding Strichartz estimates [9]

$$
\int_{0}^{1} \int_{-\infty}^{+\infty} \int_{-\infty}^{+\infty}|T(z) w|^{4} d x d y d z \leqslant C P(w)^{2} \leqslant C P^{2} .
$$

Carrying out the same calculations, one can obtain averaged ground states in a one-dimensional NLS with critical (quintic) nonlinearity

$$
i u_{Z}+\left[1+\frac{1}{\epsilon} d\left(\frac{Z}{\epsilon}\right)\right] u+a|u|^{2} u+b|u|^{4} u=0
$$

with $a \geqslant 0, b>0$.

Now we present some results of numerical simulations that confirm the possibility of finding ground states in critical NLS equations. We have simulated the original evolutionary equations (3) and (4) with a variable coefficient $d(z)$ that was chosen to be \pm 1 on $z \in[0,0.5]$ and $z \in[0.5,1]$, respectively, and with $\epsilon=0.1$.

Since it is difficult to create ground states both numerically and experimentally, we followed the approach in [4] of approximating ground states with Gaussian pulses. More precisely, we found a Gaussian pulse that minimizes the averaged Hamiltonian, subject to the power constraint. An approximating Gaussian pulse takes the form $w(x, y)$ $=A \exp \left(r^{2} / \sigma\right)$, where $A$ is a real amplitude, $\sigma$ is a complex parameter, and $r$ is the Euclidean distance. Substituting this 


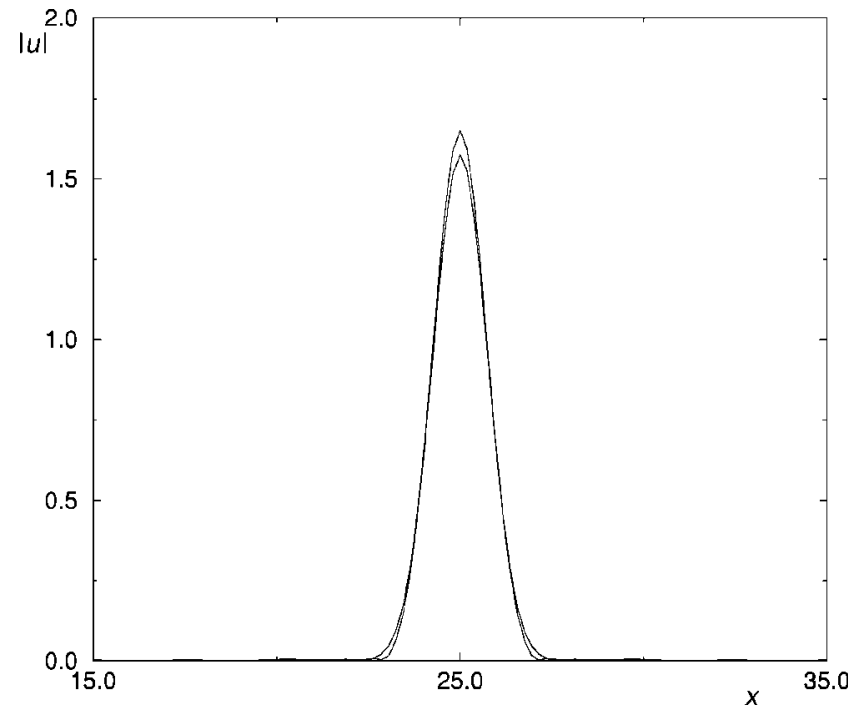

FIG. 1. Spatial dispersion-managed soliton at $t=0$ and $t$ $=400\left(\epsilon=0.1, D_{0}=0.5\right)$.

function into the expression for the corresponding averaged Hamiltonian and using the power relation, after lengthy but straightforward calculations, we obtain a function in one complex variable $\sigma$ which must be minimized. The real amplitude $A$ is then found from the power relation.

The initial pulse profiles obtained in this way have been used in numerical simulations. The simulations show that stabilization does take place (see Figs. 1-4). Indeed, after $t$ $=400$ an arbitrary pulse would at least double its width (our simulations confirm this too), while specially prepared pulses only slightly deform (Figs. 1 and 3). Amplitude oscillations (see Figs. 2 and 4) also indicate that the pulse solutions are near the minimum of the Hamiltonian functional. The oscillations are due to the fact that no Gaussian pulse is exactly a ground state and therefore the solution oscillates near the ground state.

In conclusion, we have demonstrated the existence and stability of a periodic DM soliton. We have shown that the averaged equation possesses a ground state that minimizes

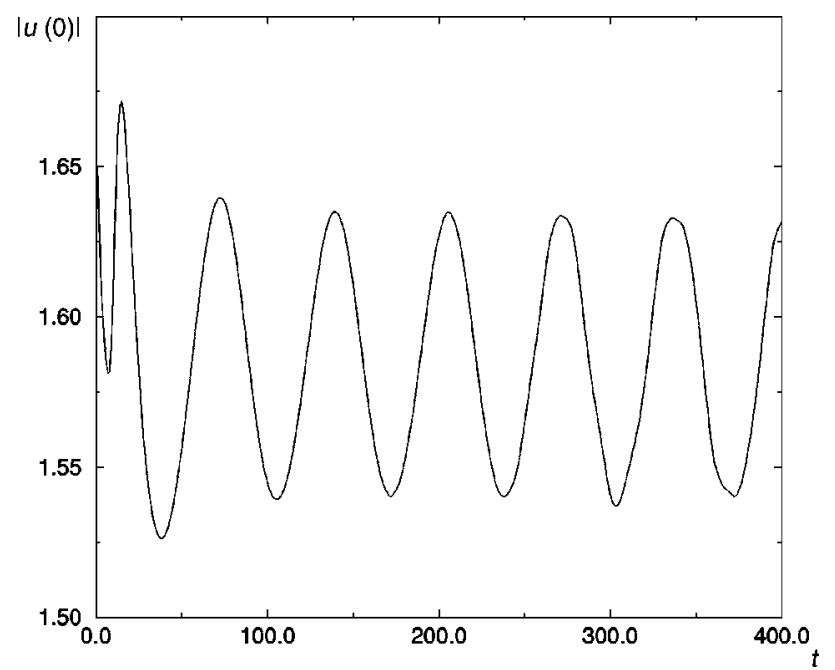

FIG. 2. Amplitude variation of spatial dispersion-managed soliton.

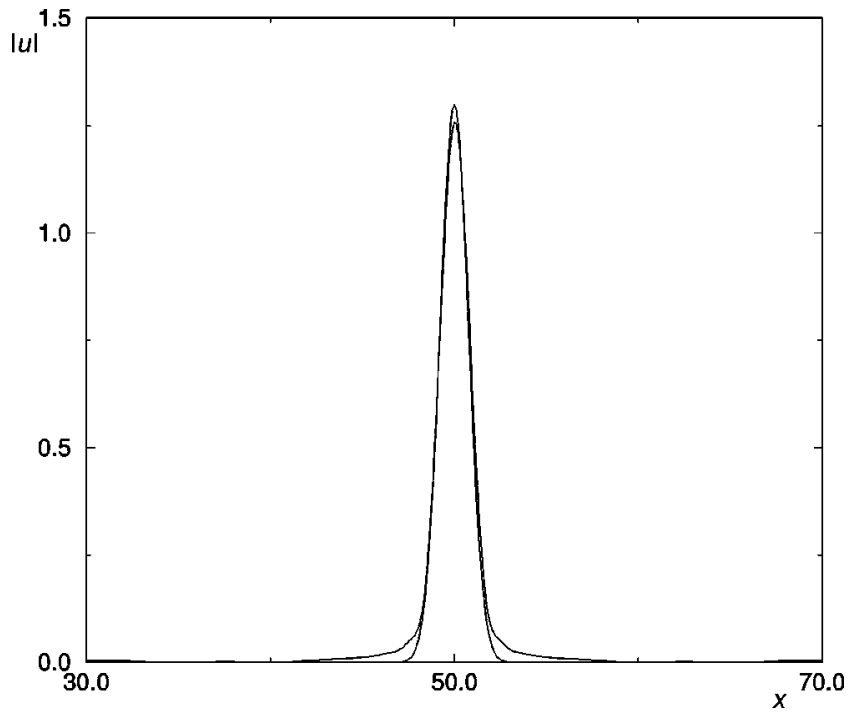

FIG. 3. Dispersion-managed soliton in quintic NLS at $t=0$ and $t=800\left(\epsilon=0.1, D_{0}=1\right)$.

an averaged action functional. The corresponding solutions of the original equation are constructed from the above ground state and turn out to be nearly periodic for sufficiently long distances (while the averaging procedure remains valid).

We also found an application of the dispersion-managed soliton phenomenon that is possible due to interpretation of the phenomenon as a "dispersive", stabilization mechanism. It can also be considered as an infinite-dimensional analog of Kapitza's effect of the stabilization of an inverted pendulum. Our theoretical predictions have been confirmed with numerical simulations. This approach can also be used to investigate the possibility of stabilizing pulses in other practical systems.

\section{APPENDIX}

\section{Concentration compactness principle}

Here, we show that from any minimizing sequence of the constrained variational principle a converging subsequence

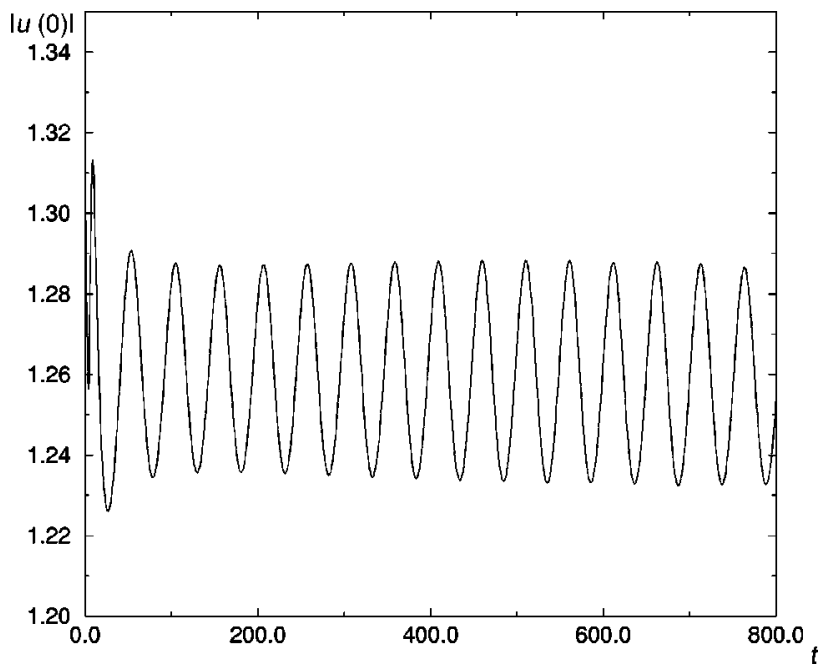

FIG. 4. Amplitude variation of dispersion-managed soliton in quintic NLS. 
can be constructed. Although the proof of this result is quite technical, it seems to be at the heart of the phenomenon of dispersion-managed solitons. The main difficulty is to show that the solutions of the linear Schrödinger equation cannot spread out in finite time if the initial data were localized. Below, we provide a heuristic explanation of this result.

It turns out that a minimizing sequence $u_{m}$ such that $G\left(u_{m}\right)$ is bounded and $P\left(u_{m}\right)=\lambda$ must have a subsequence $u_{m_{k}}$ (which we will denote by $u_{k}$ ) for which one of the following possibilities occurs [11]. (1) It converges to a localized pulse $u$ [satisfying $P(u)=\lambda$ ] up to translations $\tau_{k}$, i.e., $G\left(w_{k}-u\right) \rightarrow 0$, where $w_{k}(\tau)=u_{k}\left(\tau-\tau_{k}\right)$. (2) It is vanishing, i.e., $\sup _{y \in R^{1}} \int_{y-1}^{y+1}\left|u_{k}\right|^{2} d \tau \rightarrow 0$. (3) It splits into two parts, i.e., for some $\gamma(0<\gamma<\lambda)$ and any $\epsilon>0$ there are two subsequences $v_{k}$ and $w_{k}$ such that $P\left(u_{k}-v_{k}-w_{k}\right)<\epsilon, P\left(v_{k}\right)$ $=\gamma, P\left(w_{k}\right)=\lambda-\gamma$, and $\operatorname{dist}\left(\operatorname{supp}\left(v_{k}\right), \operatorname{supp}\left(w_{k}\right)\right) \rightarrow \infty$.

Our goal is to show that the second and third possibilities cannot occur. The main reason for that is the subadditivity of the constraint minimization problem, $P_{\lambda_{1}+\lambda_{2}}<P_{\lambda_{1}}+P_{\lambda_{2}}$, which can be verified by a scaling argument. This implies that in order to make $\langle H\rangle\left(u_{k}\right) \rightarrow P_{\lambda}$ the sequence should be "tight." Indeed, imagine that $u_{k}$ splits as in (3), so that $\langle H\rangle\left(v_{k}+w_{k}\right)=\langle H\rangle\left(v_{k}\right)+\langle H\rangle\left(w_{k}\right) \quad$ with $\quad P\left(v_{k}\right)=\gamma \quad$ and $P\left(w_{k}\right)=\lambda-\gamma$. Then the properties of the infimum and the subadditivity condition suggest that

$$
\langle H\rangle\left(v_{k}+w_{k}\right)>P_{\gamma}+P_{\lambda-\gamma}>P_{\lambda},
$$

and therefore the infimum cannot be attained.

We first show that vanishing does not occur. By the definition of the minimizing sequence, we have for sufficiently large $k$

$$
\int_{0}^{1} \int_{-\infty}^{+\infty}\left|T(z) u_{k}\right|^{4} d \tau d z \geqslant c>0
$$

which implies that for some $z_{0}$

$$
\int_{-\infty}^{+\infty}\left|T\left(z_{0}\right) u_{k}\right|^{4} d \tau \geqslant c>0
$$

Applying the estimate obtained by Cazenave [9],

$$
\int_{-\infty}^{+\infty}|u|^{4} d \tau \leqslant C_{1} \sup _{y \in R^{1}} \int_{y-1}^{y+1}|u|^{2} d \tau G(u)^{2},
$$

to $T\left(z_{0}\right) u_{k}$ we can conclude that

$$
\sup _{y \in R^{1}} \int_{y-1}^{y+1}\left|T\left(z_{0}\right) u_{k}\right|^{2} d \tau \geqslant c_{0},
$$

where $c_{0}$ is independent of $k$. Now we assume that our sequence is vanishing and show that it leads to a contradiction. For definiteness, we assume that $y=0$ (which corresponds to centering the sequence) and $d(z)=1$ on $0 \leqslant z \leqslant z_{0} .{ }^{1}$ The second assumption implies that for $0 \leqslant z \leqslant z_{0} \quad u(z, \tau)$

\footnotetext{
${ }^{1}$ The general case with $d(z)$ having a finite number of nondegenerate zeros can be reduced to the one we consider.
}

$=T(z) u(0, \tau)$ is a solution of the linear Schrödinger equation $i u_{z}+u_{\tau \tau}=0$, which implies the following energy relation:

$$
\frac{d}{d z} \int_{-R}^{+R}\left|u_{k}(\tau, z)\right|^{2} d \tau=2 \operatorname{Im}\left[u_{k}(\tau, z) \frac{\partial u_{k}}{\partial \tau}(\tau, z)\right]_{-R}^{+R} .
$$

Integrating the above equation over $z$ we obtain

$$
\begin{array}{r}
\int_{-R}^{+R}\left|u_{k}\left(\tau, z_{0}\right)\right|^{2} d \tau-\int_{-R}^{+R}\left|u_{k}(\tau, 0)\right|^{2} d \tau \\
=\int_{0}^{z_{0}} 2 \operatorname{Im}\left[u_{k}(\tau, z) \frac{\partial u_{k}}{\partial \tau}(\tau, z)\right]_{-R}^{+R} d z
\end{array}
$$

But the left hand side can be estimated from

$$
\begin{aligned}
c_{0}-2(R+1) \epsilon_{k} \leqslant & \int_{-R}^{+R}\left|u_{k}(z, \tau)\right|^{2} d x \\
& -\int_{-R}^{+R}\left|u_{k}(\tau, 0)\right|^{2} d \tau \\
= & \int_{0}^{z_{0}} 2 \operatorname{Im}\left[u_{k}(z, \tau) \frac{\partial u_{k}}{\partial \tau}(z, \tau)\right]_{-R}^{+R} d z
\end{aligned}
$$

where

$$
\boldsymbol{\epsilon}_{k}=\sup _{y \in R^{1}} \int_{y-1}^{y+1}\left|u_{k}\right|^{2} d \tau \text {. }
$$

Integrating this inequality over $R$, we obtain

$$
\begin{aligned}
& \int_{0}^{R_{0}}\left[c_{0}-2(R+1) \epsilon_{k}\right] d R \\
& \quad \leqslant 2 \int_{-R_{0}}^{+R_{0}} \int_{0}^{z_{0}}\left|u_{k}(R, z)\right|\left|\frac{\partial u_{k}}{\partial \tau}(R, z)\right| d z d R,
\end{aligned}
$$

where $R_{0}$ is the solution of the equation $c_{0}-2(R+1) \epsilon_{k}$ $=0$. Therefore

$$
\begin{aligned}
\frac{C}{\epsilon_{k}} & \leqslant 2 \int_{-R_{0}}^{+R_{0}} \int_{0}^{z_{0}}\left|u_{k}(R, z)\right|\left|\frac{\partial u_{k}}{\partial \tau}(R, z)\right| d z d R \\
& \leqslant 2 \int_{-\infty}^{+\infty} \int_{0}^{z_{0}}\left|u_{k}(R, z)\right|\left|\frac{\partial u_{k}}{\partial \tau}(R, z)\right| d z d R \\
& \leqslant 2 \int_{0}^{z_{0}}\left\|u_{k}\right\|_{L^{2}(R)} \|\left.\frac{\partial u_{n}}{\partial \tau}\right|_{L^{2}(R)} d z \leqslant C z_{0} .
\end{aligned}
$$

Taking $k \rightarrow \infty$ so that $\epsilon_{k} \rightarrow 0$ we obtain a contradiction.

Similarly, we can show that splitting does not occur. Indeed, if it did, then taking $u_{m_{k}}=v_{k}+w_{k}+h_{k}$, where $h_{k}$ is small, we obtain

$$
\langle H\rangle\left(u_{m_{k}}\right)=\langle H\rangle\left(v_{k}\right)+\langle H\rangle\left(w_{k}\right)+R_{k},
$$

where $R_{k}$ represents the terms that are vanishing as $\epsilon \rightarrow 0$. This can be shown by applying the above argument. Therefore using the subadditivity condition $P_{\lambda_{1}+\lambda_{2}}<P_{\lambda_{1}}+P_{\lambda_{2}}$, 
we obtain a contradiction, as explained above, with the fact that $u_{m_{k}}$ is a minimizing sequence.

\section{Negativity of the infimum}

We construct a family $u_{\lambda}$ satisfying the constraint $P\left(u_{\lambda}\right)=\lambda$ so that $\langle H\rangle\left(u_{\lambda}\right)<0$. We start with a Gaussian ansatz

$$
u(x)=A e^{x^{2} / 2 \sigma},
$$

which turns out to be a self-similar solution of the linear Schrödinger equation

$$
i u_{t}+d(t) u_{x x}=0 \text {. }
$$

Indeed, substituting Eq. (A1) in Eq. (A2) we obtain

$$
i \dot{A}+d(t) \frac{A}{\sigma}=0, \quad i 2 \dot{\sigma}-2 d(t)=0 .
$$

Solving this system of ordinary differential equations, we obtain

$$
u(x, t)=\frac{A_{0} \sqrt{\sigma_{0}}}{\sqrt{\sigma_{0}-i 2 D(t)}} e^{x^{2} / 2\left[\sigma_{0}-i 2 D(t)\right]},
$$

where $\dot{D}(t)=d(t)$ and $D(0)=0$. Now we evaluate the averaged functional and the constraint over the self-similar solutions obtained,

$$
\begin{aligned}
P(u) & =\int_{-\infty}^{+\infty}|u(x, t)|^{2} d x \\
& =|A(t)|^{2} \int_{-\infty}^{+\infty} e^{x^{2} \operatorname{Re}[\sigma(t)] /|\sigma(t)|^{2} d x} \\
& =\frac{|A(t)|^{2}|\sigma(t)|}{\sqrt{|\operatorname{Re}[\sigma(t)]|}} \sqrt{\pi}=\frac{\left|A_{0}\right|^{2}\left|\sigma_{0}\right|}{\sqrt{|\operatorname{Re}[\sigma(t)]|}} \sqrt{\pi},
\end{aligned}
$$

where we have assumed that $\operatorname{Re}\left(\sigma_{0}\right)<0$. After straightforward calculations, we also obtain

$$
\begin{aligned}
Q(u) & =\int_{0}^{1} \int_{-\infty}^{+\infty}\left|u_{x}(x, t)\right|^{2} d x d t \\
& =\frac{|A(t)|^{2}|\sigma(t)|}{\sqrt{\mid \operatorname{Re}[\sigma(t)]^{3}}} \frac{\sqrt{\pi}}{2}=\frac{\lambda}{2|\operatorname{Re}[\sigma(t)]|}=\frac{\lambda}{2\left|\operatorname{Re}\left(\sigma_{0}\right)\right|},
\end{aligned}
$$

where we have used Eq. (A3) and the time independence of $Q$ for the solutions of the linear Schrödinger equations. Finally, using Eq. (A3), we calculate

$$
\begin{aligned}
\int_{0}^{1} \int_{-\infty}^{+\infty}|T u|^{4} d x d t & =\int_{0}^{1} \int_{-\infty}^{+\infty}|A(t)|^{4} e^{x^{2} 2 \operatorname{Re}[\sigma(t)] /|\sigma(t)|^{2}} d x d t \\
& =\int_{0}^{1} \frac{|A(t)|^{4}|\sigma(t)|}{\sqrt{2|\operatorname{Re}[\sigma(t)]|}} \sqrt{\pi} d t \\
& =\frac{\lambda^{2}}{\sqrt{2 \pi}} \int_{0}^{1} \frac{\sqrt{|\operatorname{Re}[\sigma(t)]|}}{|\sigma(t)|} d t
\end{aligned}
$$

Now, we evaluate the averaged functional

$$
\begin{aligned}
\langle H\rangle(u)= & \int_{-\infty}^{+\infty} \int_{0}^{1}\left(\alpha\left|u_{x}\right|^{2}-\frac{1}{2}|T(t) u|^{4}\right) d x d t \\
= & \frac{\alpha \lambda}{2\left|\operatorname{Re}\left(\sigma_{0}\right)\right|}-\frac{\lambda^{2}}{2 \sqrt{2 \pi}} \int_{0}^{1} \frac{\sqrt{|\operatorname{Re}[\sigma(t)]|}}{|\sigma(t)|} d t \\
= & \frac{\lambda \alpha}{2\left|\operatorname{Re}\left(\sigma_{0}\right)\right|}\left[1-\frac{\lambda \sqrt{\left|\operatorname{Re}\left(\sigma_{0}\right)\right|^{3}}}{\alpha \sqrt{2 \pi}}\right. \\
& \left.\times \int_{0}^{1} \frac{d t}{\sqrt{\left[\operatorname{Re}\left(\sigma_{0}\right)\right]^{2}+\left[\operatorname{Im}\left(\sigma_{0}\right)-2 D(t)\right]^{2}}}\right] .
\end{aligned}
$$

Now it is easy to see that for any fixed $\alpha>0$ and arbitrarily small $\lambda$ we can take $\operatorname{Im}\left(\sigma_{0}\right)=0$ and sufficiently large $\operatorname{Re}\left(\sigma_{0}\right)$ so that the averaged functional will become negative, $\langle H\rangle(u)<0$.
[1] A.F. Evans, in Optical Fiber Communication Conference, Vol. 2 of OSA Technical Digest Series (OSA, Washington, D.C., 1998), p. 22.

[2] C. Lin, H. Kogelnik, and L.G. Cohen, Opt. Lett. 5, 476 (1980).

[3] S.K. Turitsyn, E.G. Shapiro, and V.K. Mezentsev, Opt. Fiber Technol.: Mater., Devices Syst. 4, 384 (1998); S.K. Turitsyn, T. Shaefer, K.H. Spatschek, and V.K. Mezentsev, Opt. Commun. 163, 122 (1999).

[4] I. Gabitov and S.K. Turitsyn, Opt. Lett. 21, 327 (1996); Pis'ma Zh. Éksp. Teor. Fiz. 63, 814 (1996) [JETP Lett. 63, 861 (1996)].

[5] S. Schochet, J. Diff. Eqns. 114, 476 (1994).
[6] E. Grenier, J. Math. Pures Appl. 76, 477 (1997).

[7] E. Grenier, C.K.R.T. Jones, S. Turitsyn, and V. Zharnitsky, Physica D (to be published).

[8] S.K. Turitsyn and V.K. Mezentsev, Pis'ma Zh. Éksp. Teor. Fiz. 68, 791 (1998) [JETP Lett. 68, 830 (1998)].

[9] T. Cazenave, An Introduction to Nonlinear Schrödinger Equations (Universidade Federal do Rio de Janeiro, Instituto de Matematica, Rio de Janeiro, 1993).

[10] L.D. Landau and E.M. Lifshitz, Mechanics (Pergamon Press, Oxford, 1960).

[11] P.-L. Lions, Rev. Mat. Iberoam. 1, 45 (1985). 